\title{
Adição de farinha de resíduos de guavira em barra de cereais: aceitabilidade sensorial e caracterização físico- química
}

\section{Addition of guavira residue flour in cereal bar: physical-chemical characterization and sensory acceptability between children}

Jessica Patrícia Cain ${ }^{1}$, Ana Carolina Gaspar da Silva ${ }^{1}$, Jaqueline Machado Soares ${ }^{1}$, Mirelly Marques Romeiro Santos ${ }^{2}$, Luane Aparecida do Amaral ${ }^{2}$, Elisvânia Freitas dos Santos ${ }^{2}$, Daiana Novello ${ }^{1}$

${ }^{1}$ Universidade Estadual do Centro-Oeste (UNICENTRO), Guarapuava, Paraná, Brasil.

${ }^{2}$ Universidade Federal de Mato Grosso do Sul (UFMS), Campo Grande, Mato Grosso do Sul, Brasil.

\begin{abstract}
Resumo
Introdução: A guavira é uma fruta nativa do cerrado brasileiro. Geralmente, a polpa é utilizada pela indústria na elaboração de produtos alimentícios, contudo seus resíduos são descartados. O uso de subprodutos pode ser uma estratégia para melhorar o aporte nutricional de alimentos normalmente consumidos, além de reduzir o lixo orgânico depositado no meio ambiente. Objetivos: Avaliar a aceitabilidade sensorial de barra de cereais adicionada de diferentes níveis de farinha de resíduos de guavira (FRG) entre crianças. Também, determinar a composição físico-química do produto padrão e daquele com maior teor de FRG e aceitação semelhante ao produto padrão. Metodologia: Foram elaboradas 6 formulações de barra de cereais: F1: padrão ( $0 \%$ de FRG) e as demais adicionadas de $2 \%$ (F2), 4\% (F3), 6\% (F4), 8\% (F5) e 10\% (F6) de FRG. Participaram da avaliação sensorial 62 julgadores não treinados, com idade entre 7 a 10 anos. Resultados: Maiores notas para os atributos de aparência, aroma, sabor, cor e para a aceitação global e intenção de compra foram verificadas para a formulação padrão quando comparada a F6. As demais amostras não apresentaram diferença significativa $(\mathrm{p}>0,05)$ A textura não foi influenciada pela adição de FRG. Assim, a formulação F5 foi considerada aquela com maior teor de FRG (8\%) e com aceitação sensorial similar ao produto padrão. Maiores teores de cinzas, lipídio, carboidrato, energia e fibras foram verificados para F5 quando comparada à formulação padrão. Conclusão: Um nível de adição de até $8 \%$ de FRG em barra de cereais é bem aceito pelas crianças, além de aumentar os teores de fibras e minerais do produto.
\end{abstract}

Palavras-chave: Aceitabilidade; Fibra dietética; Subprodutos.

Autor correspondente:

Daiana Novello

Endereço: Rua Simeão Camargo Varela de Sá, 03, Vila Carli

CEP: 85040-080, Guarapuava, Paraná, Brasil.

E-mail: nutridai@gmail.com
Recebido em: 06/11/2018 Revisado em: 23/11/2018 Aceito em: 24/05/2019 


\begin{abstract}
Introduction: Guavira is a native fruit of the Brazilian cerrado. Generally, pulp is used by the industry in the elaboration of food products, but its residues are discarded. The use of by-products can be a strategy to improve the nutritional supply of foods normally consumed, besides reducing the organic waste deposited in the environment. Objectives: To evaluate the sensory acceptability of cereal bars added to different levels of guavira residue flouer (GRF) among children. Also, determine the physicochemical composition of the standard product and the one with the highest GRF content and similar acceptance to the standard product. Methodology: Six formulations of cereal bar were prepared: F1: standard (0\% GRF) and the others added 2\% (F2), 4\% (F3), 6\% (F4), 8\% (F5) and $10 \%$ (F6) of GRF. A total of 62 untrained judges aged 7 to 10 years participated in the sensory evaluation. Results: Higher grades for the appearance, aroma, taste, color attributes and for overall acceptance and purchase intention were checked for the standard formulation when compared to F6. The other samples had no significant difference $(p>0.05)$. The texture was not influenced by the addition of GRF. Thus, the formulation F5 was considered the one with the highest GRF content (8\%) and with sensorial acceptance similar to the traditional product. Higher ash, lipid, carbohydrate, energy and fiber contents were verified for F5 when compared to the standard formulation. Conclusion: A level of up to $8 \%$ addition of GRF in cereal bars is well accepted by children, and besides increasing the fiber content and mineral of the product.
\end{abstract}

Keywords: Acceptability; Dietary Fiber; By-products.

\section{Introdução}

O desperdício de alimentos ocorre de diferentes formas ao longo da cadeia produtiva. As fases de produção e de consumo são as principais responsáveis, uma vez que representam $56 \%$ do total de perdas no mundo. Estima-se que 1,3 bilhões de toneladas de alimentos sejam anualmente desperdiçadas, destas $55 \%$ são provenientes de frutas e hortaliças1. Dessa forma, o uso de resíduos alimentares como ingredientes em preparações pode ser uma estratégia para a indústria de alimentos, já que pode diminuir a quantidade descartada na natureza2. Além disso, o uso de subprodutos pode melhorar o perfil nutricional dos produtos. As partes não convencionais dos alimentos, como cascas, sementes e bagaço de frutas, apresentam elevadas concentrações de nutrientes e compostos bioativos, que incluem vitaminas, minerais, fibras, fenólicos e pigmentos como antocianinas e carotenoides. Esses compostos apresentam, atividades antibacterianas, antitumorais, antivirais, antimutagênicas e cardioprotetoras, o que justifica o interesse constante do uso de subprodutos de frutas para a produção de alimentos funcionais e nutracêuticos3,4,5. Estudos realizados com farinha de cascas de goiaba6, de uva7 e de abacaxi8 demonstraram boa aceitação sensorial, alto potencial de comercialização e melhora no teor nutricional, especialmente com o aumento de fibra alimentar.

A guavira (Campomanesia adamantium), também conhecida como gabiroba, guabiroba ou guariroba, é uma planta nativa do cerrado brasileiro. Os frutos crescem em pequenos arbustos e possuem cores vivas (verde, amarelo, laranja e roxo), aroma cítrico, sabor marcante e teores vitamínicos consideráveis9,10. O período de colheita da guavira varia de novembro a dezembro, mas a alta perecibilidade, juntamente com a falta de tratamento pós-colheita, são fatores que dificultam o consumo e a conservação in natura, contribuindo para o desperdício. Geralmente, a polpa da guavira é utilizada pela indústria como aromatizante em licores e sucos e na produção de sorvetes, doces, molhos e mousses 9,11 . No entanto, os resíduos são, quase sempre, descartados, apesar de possuírem elevados teores de fibra alimentar $(19,25$ g.100g-1), de vitamina C $(218,24 \mathrm{mg} .100 \mathrm{~g}-1)$, de proteína $(3,12$ g. $100 \mathrm{~g}-1)$ e de lipídio $(3,65 \mathrm{~g} .100 \mathrm{~g}$ 1)12. Assim os resíduos são indicados para adição como ingrediente em produtos alimentícios, visando o enriquecimento nutricional. No estudo de Cristofel13, a adição de resíduos da guavira em hambúrguer de tilápia aumentou o teor de umidade e de fibras do produto final, apresentando boa aceitação sensorial. Além disso, não alterou características como o pH, atividade de água e rendimento na cocção. Houve alteração na cor do produto, principalmente pela presença de carotenoides $(0,77 \mu \mathrm{g} .100 \mathrm{~g}-1) 14$. Não há evidências científicas com o uso de farinha de resíduos de guavira em produtos à base de cereais. Isso demonstra a necessidade de pesquisas que avaliem sua utilização como ingrediente em novos alimentos, como as barras de cereais, que são de fácil preparo e apresentam elevada aceitabilidade por diferentes públicos 15,16 .

Atualmente, a indústria alimentícia está direcionando maiores investimentos na elaboração de produtos que atendam às necessidades das crianças, como é o caso das barras de cereais. Esse produto tem
Comentado [A2]: Todos os autores do texto foram renumerados 
Adição de farinha de resíduos de guavira

mostrado um rápido crescimento no mercado, o que se deve, também, a sua praticidade e facilidade de consumo17,18. Representam uma alternativa de complemento alimentar, comercializada em diferentes tipos, sabores e composição nutricional. Em geral, as barras de cereais contêm teores consideráveis de fibras, contudo podem apresentar quantidades elevadas de sódio, de gorduras e de açúcar19. Assim, quando consumidas em excesso, podem colaborar com o desenvolvimento de doenças crônicas não transmissíveis, como o sobrepeso e a obesidade desde a infância20.

No caso de crianças em idade escolar (7-10 anos), a escolha de um produto alimentício para a compra está associada principalmente aos aspectos sensoriais21. Dessa forma, é imprescindível o uso de ferramentas que possam auxiliar na caracterização do produto, visando sua aceitabilidade no mercado22. A análise sensorial é um método utilizado para analisar as sensações percebidas pelos consumidores referentes a um alimento. Essa avaliação é baseada nos cincos sentidos humanos (visão, audição, paladar, olfato e tato), o que permite uma compreensão global do produto desenvolvido23. Além disso, análises físicoquímicas são necessárias para identificar a qualidade e a composição do produto, bem como as possíveis transformações que podem ocorrer durante o processamento. Isso, possibilita a adequação dos produtos às normas de comercialização exigidas pelo país de origem, além de garantir a segurança alimentar ao consumidor final22. Sabendo disso, os objetivos do trabalho foram verificar a aceitabilidade sensorial de barra de cereais adicionada de farinha de resíduos de guavira (FRG) entre crianças, determinar a composição físico-química da formulação padrão e daquela com maior teor de FRG e aceitação sensorial semelhante ao produto padrão.

\section{Metodologia}

Matéria-prima e elaboração da farinha de resíduos de guavira

Foram utilizados resíduos (casca, semente, pedúnculo) de guavira provenientes da indústria de polpas de guavira (Reserva Ouro Verde) do Município de Miranda, Mato Grosso do Sul. O preparo da FRG se deu no Laboratório de Processamento de Alimentos da Unidade de Tecnologia de Alimentos e Saúde Pública (UTASP/UFMS) da Universidade Federal de Mato Grosso do Sul (UFMS). A FRG foi obtida a partir da desidratação dos resíduos em estufa (Lawes ${ }^{\circledR}$, Brasil), com circulação de ar $\left(40{ }^{\circ} \mathrm{C}\right)$ durante 24 horas. Após a secagem, os resíduos foram fragmentados em liquidificador industrial (FAK ${ }^{\circledR}$, Brasil) e passados em peneira (Tamis $20 \mathrm{Mesh}$ ). A FRG teve um rendimento de $25 \%$ de material pulverizado, transportada sob refrigeração $\left(10^{\circ} \mathrm{C}\right)$ até o município de Guarapuava, PR, onde foram realizadas as análises sensoriais e físico-químicas. Os demais ingredientes utilizados na barra de cereais foram adquiridos em supermercados localizados nesta cidade.

\section{Formulações}

Foram elaboradas 6 formulações de barra de cereais: F1: padrão (0\% de FRG) e as demais adicionadas de $2 \%(\mathrm{~F} 2), 4 \%(\mathrm{~F} 3), 6 \%(\mathrm{~F} 4), 8 \%(\mathrm{~F} 5) \mathrm{e}$ $10 \%$ (F6) de FRG. Essas porcentagens foram definidas por meio de testes sensoriais preliminares realizados com o produto. Além da FRG, os ingredientes utilizados nas formulações foram: banana caturra $(20,53 \%)$, farinha de trigo (F1: $14 \%, \mathrm{~F} 2: 12 \%, \mathrm{~F} 3$ 10\%, F4: $8 \%$, F5: 6\%, F6: 4\%), aveia em flocos $(13,44 \%)$, granola $(12,32 \%)$, uva passa $(10,95 \%)$, farinha de linhaça $(10,27 \%)$, castanha do Pará $(6,84 \%)$, açúcar demerara orgânico $(6,84 \%)$, mel $(4,11 \%)$ e canela $(0,68 \%)$.

As formulações foram preparadas individualmente misturando-se todos os ingredientes até completa homogeneização. A massa foi moldada e cortada em retângulos com tamanho aproximado de $2 \mathrm{~cm}$ altura $x$ $3 \mathrm{~cm}$ comprimento $\mathrm{x} 1 \mathrm{~cm}$ espessura. As formulações foram assadas em forno semi-industrial (Venâncioß, Brasil), pré-aquecido a $250{ }^{\circ} \mathrm{C}$ por 10 minutos. Após esse processo, permaneceram em repouso até atingirem a temperatura ambiente $\left(22^{\circ} \mathrm{C}\right)$. Em seguida, foram acondicionadas em recipiente plástico hermeticamente fechado até o momento da análise sensorial, que ocorreu em 24 horas posteriores ao preparo.

\section{Análise sensorial}

De acordo com os critérios estabelecidos pela Resolução $\mathrm{n}^{\circ} 26$ do Fundo Nacional de Desenvolvimento da Educação24, quando ocorrerem quaisquer alterações inovadoras ou quando deseja-se inserir um alimento atípico no hábito alimentar local, faz-se necessário a aplicação de testes sensoriais. Com base nisso, participaram da pesquisa sensorial 62 julgadores não treinados, de ambos os gêneros, com idade entre 7 a 10 anos. As crianças participantes estavam devidamente matriculadas em uma escola municipal de Guarapuava, PR, escolhida por conveniência. Os produtos foram avaliados sensorialmente em uma sala da escola, conforme indicação da diretora. Cada prova foi feita individualmente, e o julgador foi orientado pelas pesquisadoras sem interferência nas respostas. Foram avaliados atributos de aparência, aroma, sabor, textura e cor, por meio de uma escala hedônica facial estruturada mista de 7 pontos variando de 1 (super ruim) a 7 (super bom). Também, foram aplicadas questões de aceitação global e intenção de compra, analisadas com o uso de uma escala estruturada de 5 pontos (1 - desgostei muito/não compraria a 5 - gostei muito/compraria com certeza)23. Os julgadores receberam uma porção de cada amostra 
Adição de farinha de resíduos de guavira

aproximadamente $15 \mathrm{~g}$ ), em guardanapos brancos descartáveis, codificados com números de três dígitos, de forma casualizada e balanceada, acompanhadas de um copo de água para limpeza do palato. As formulações foram oferecidas aos julgadores de forma monádica sequencial. O cálculo do Índice de Aceitabilidade (IA) foi realizado conforme a fórmula: IA $(\%)=\mathrm{A} \times 100 / \mathrm{B}(\mathrm{A}=$ nota média obtida para $\mathrm{o}$ produto e $\mathrm{B}=$ nota máxima dada ao produto) 25 .

\section{Caracterização físico-química}

As seguintes análises físico-químicas foram realizadas em triplicata na FRG, na formulação padrão e naquela com maior nível de adição de FRG e com aceitação sensorial semelhante ao produto padrão: Umidade: determinada em estufa a $105{ }^{\circ} \mathrm{C}$ até peso constante; Cinzas: analisadas em mufla $\left(550{ }^{\circ} \mathrm{C}\right) 27$; Lipídio: utilizou-se o método de extração a frio26; Proteína: avaliada por meio do teor de nitrogênio total da amostra, pelo método Kjeldahl, determinado ao nível semimicro. Utilizou-se o fator de conversão de nitrogênio para proteína de 6,25; Fibra alimentar: Fibra alimentar total e insolúvel, determinadas por método enzimático27. O teor de fibra alimentar solúvel foi calculado pela diferença dos resultados de fibra alimentar total e insolúvel; Carboidrato: avaliado por cálculo teórico (por diferença) nos resultados das triplicatas, conforme a fórmula: $\%$ Carboidrato $=100$ - $(\%$ umidade $+\%$ proteína $+\%$ lipídio $+\%$ cinzas + $\%$ fibra); Valor calórico total (kcal): calculado utilizando-se os seguintes valores: lipídios $(8,37$ $\mathrm{kcal} / \mathrm{g})$, proteína $(3,87 \mathrm{kcal} / \mathrm{g})$ e carboidratos $(4,11$ $\mathrm{kcal} / \mathrm{g}) 28$ O Valor Diário de Referência (VD): calculado em relação a $25 \mathrm{~g}$ da amostra, com base nos valores médios diários preconizados para crianças ( 7 a 10 anos)29, resultando em: $1.933,5 \mathrm{kcal} / \mathrm{dia}, 130 \mathrm{~g} / \mathrm{dia}$ de carboidrato, 26,5 g/dia de proteína e $26,75 \mathrm{~g} /$ dia de fibra alimentar.
Análise estatística

Os dados foram analisados com auxílio do software Statgraphics Plus ${ }^{\circledR}$, versão 5.1, por meio da análise de variância (ANOVA). A comparação de médias foi realizada pelo teste de médias de Tukey e de student, avaliados com nível de $5 \%$ de significância.

\section{Questões éticas}

Este trabalho foi aprovado pelo Comitê de Ética em Pesquisa da UNICENTRO, parecer número $\mathrm{n}^{\circ}$ 608.950/2014. Entretanto, como critérios de exclusão foram considerados os seguintes fatores: possuir alergia a algum ingrediente utilizado na elaboração da barra de cereais ou não entregar o Termo de Consentimento Livre e Esclarecido (TCLE) assinado pelos responsáveis legais.

\section{Resultados}

Na TABELA 1 estão descritos os resultados da avaliação sensorial da barra de cereais padrão e daquelas adicionadas de diferentes concentrações de FRG. Maiores notas para os parâmetros aparência, aroma, sabor, cor, aceitação global e intenção de compra $(\mathrm{p}<0,05)$ foram verificadas para a formulação padrão, quando comparadas com F6. Não houve diferença significativa $(p>0,05)$ para as demais amostras. A adição de FRG não influenciou na textura do produto. Além disso, as formulações apresentam IA $\geq 70 \%$ em todos os atributos. A amostra F5 foi aquela com maior teor de FRG (8\%) e com aceitação sensorial similar a padrão em todas as avaliações realizadas. Diante disso, foi selecionada para fins de comparação físico-química juntamente com a formulação padrão (F1).

TABELA 1: Escores sensoriais médios ( \pm desvio padrão) e Índice de Aceitabilidade (IA) da barra de cereais com adição de deferentes concentrações de farinha de resíduos de guavira

\begin{tabular}{|c|c|c|c|c|c|c|}
\hline Parâmetros & F1 & F2 & F3 & F4 & F5 & F6 \\
\hline Aparência & $6,06 \pm 1,20^{\mathrm{a}}$ & $5,66 \pm 1,46^{\mathrm{ab}}$ & $5,52 \pm 1,78^{\mathrm{ab}}$ & $5,27 \pm 1,69^{\mathrm{ab}}$ & $5,47 \pm 1,60^{\mathrm{ab}}$ & $5,00 \pm 1,81^{\mathrm{b}}$ \\
\hline IA (\%) & 86,57 & 80,86 & 78,86 & 75,29 & 78,14 & 71,43 \\
\hline Aroma & $6,05 \pm 1,40^{\mathrm{a}}$ & $5,71 \pm 1,12^{\mathrm{ab}}$ & $5,50 \pm 1,68^{\mathrm{ab}}$ & $5,39 \pm 1,66^{\mathrm{ab}}$ & $5,32 \pm 1,60^{\mathrm{ab}}$ & $5,19 \pm 1,82^{\mathrm{b}}$ \\
\hline IA (\%) & 86,43 & 81,57 & 78,57 & 77,00 & 76,00 & 74,15 \\
\hline Sabor & $6,26 \pm 1,13^{a}$ & $5,87 \pm 1,43^{\mathrm{ab}}$ & $5,63 \pm 1,99^{\mathrm{ab}}$ & $5,48 \pm 1,90^{\mathrm{ab}}$ & $5,49 \pm 1,73^{\mathrm{ab}}$ & $5,28 \pm 2,08^{b}$ \\
\hline IA (\%) & 89,43 & 83,86 & 80,43 & 78,29 & 78,43 & 75,43 \\
\hline Textura & $5,91 \pm 1,12^{\mathrm{a}}$ & $5,73 \pm 1,44^{\mathrm{a}}$ & $5,59 \pm 1,81^{\mathrm{a}}$ & $5,55 \pm 1,69^{\mathrm{a}}$ & $5,41 \pm 1,53^{\mathrm{a}}$ & $5,24 \pm 1,74^{\mathrm{a}}$ \\
\hline IA (\%) & 84,43 & 81,86 & 79,86 & 79,29 & 77,29 & 74,86 \\
\hline Cor & $5,95 \pm 1,14^{\mathrm{a}}$ & $5,53 \pm 1,29^{\mathrm{ab}}$ & $5,52 \pm 1,60^{\mathrm{ab}}$ & $5,48 \pm 1,49^{\mathrm{ab}}$ & $5,39 \pm 1,58^{\mathrm{ab}}$ & $5,15 \pm 1,78^{\mathrm{b}}$ \\
\hline IA $(\%)$ & 85,00 & 79,00 & 78,86 & 78,29 & 77,00 & 73,57 \\
\hline Aceitação global & $4,37 \pm 1,15^{\mathrm{a}}$ & $4,31 \pm 1,06^{\mathrm{ab}}$ & $4,08 \pm 1,39^{\mathrm{ab}}$ & $4,08 \pm 1,22^{\mathrm{ab}}$ & $3,90 \pm 1,43^{\mathrm{ab}}$ & $3,68 \pm 1,31^{\mathrm{b}}$ \\
\hline IA $(\%)^{3}$ & 87,40 & 86,20 & 81,60 & 81,60 & 78,00 & 73,60 \\
\hline Intenção de compra & $4,24 \pm 1,34^{\mathrm{a}}$ & $4,17 \pm 1,32^{\mathrm{ab}}$ & $3,89 \pm 1,58^{\mathrm{ab}}$ & $3,87 \pm 1,56^{\mathrm{ab}}$ & $3,61 \pm 1,66^{\mathrm{ab}}$ & $3,42 \pm 1,71^{\mathrm{b}}$ \\
\hline
\end{tabular}

Letras distintas na linha indicam diferença significativa pelo teste de Tukey (p<0,05); Adição de farinha de resíduos de guavira: F1: 0\%; F2: 2\%; F3: 4\%; F4: 6\%; F5: 8\%; F6: 10\%; Fonte: os autores. 
Adição de farinha de resíduos de guavira

Na FIGURA 1 está apresentada a distribuição da frequência de notas atribuídas aos diferentes parâmetros hedônicos obtidos na avaliação sensorial da barra de cereais padrão e daquelas com diferentes concentrações de FRG. Observa-se que a maioria das notas informadas pelas crianças foram iguais ou superiores a 5 , indicando que as formulações foram bem aceitas.
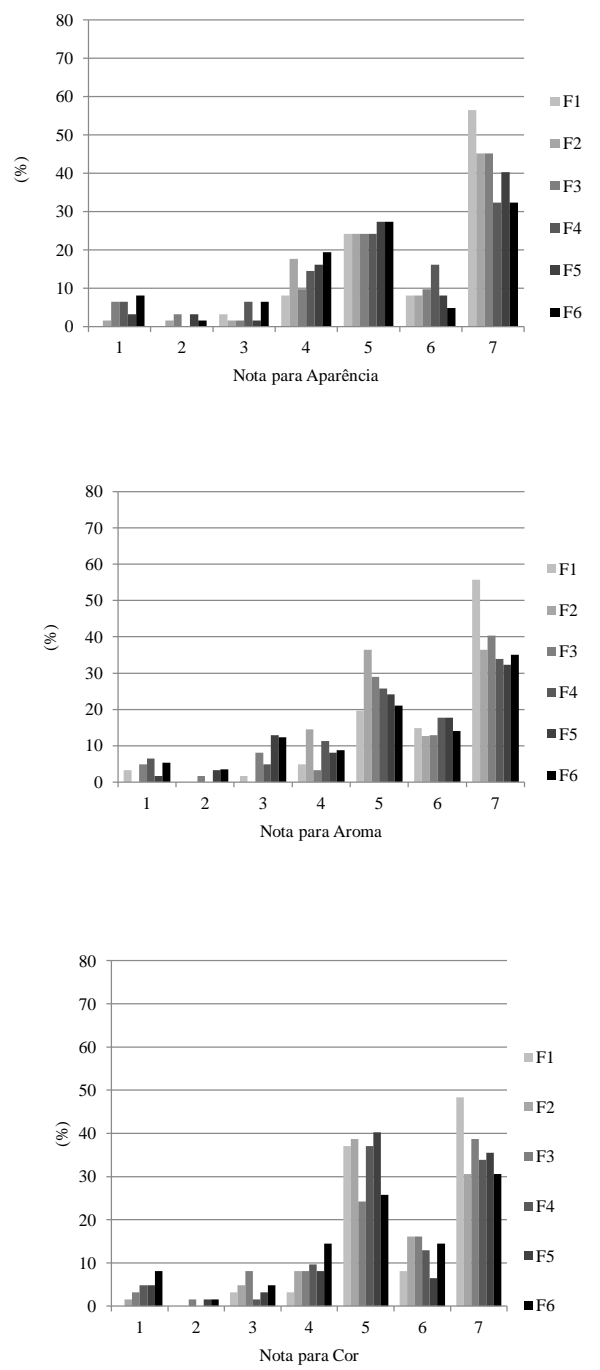
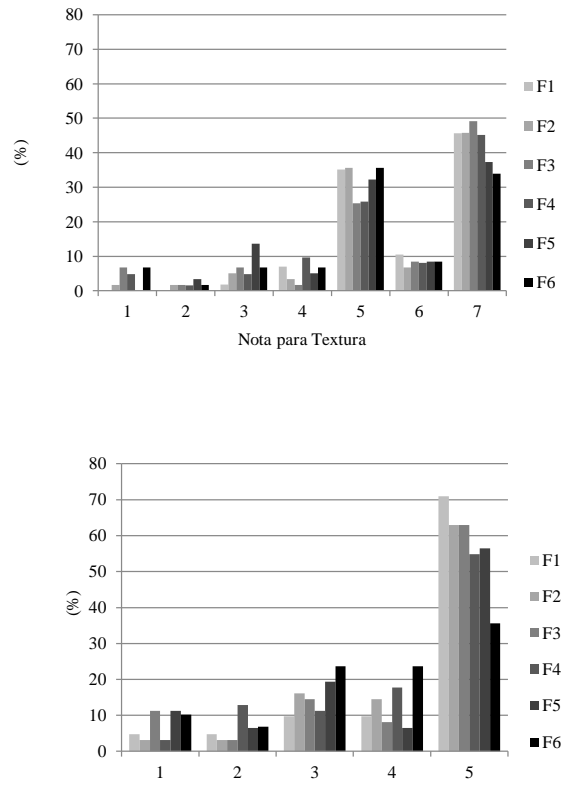

FIGURA 1: Distribuição da frequência de notas atribuída aos diferentes parâmetros hedônicos na avaliação sensorial da barra de cereais padrão e daquelas com diferentes concentrações de Farinha de Resíduos de Guavira (FRG). Fonte: os autores.

Na TABELA 2, está descrita a composição físicoquímica da FRG, da barra de cereal padrão e daquela com adição de $8 \%$ de FRG. Maiores conteúdos de umidade e proteína foram verificados em $\mathrm{F}$ 1 comparada à F5 $(\mathrm{p}<0,05)$. A amostra F5 apresentou maiores teores de cinzas, lipídio, carboidrato, energia e fibras. Destaca-se como principal resultado o teor de fibras verificado na F5, expressando um aumento de $64,5 \%$ em relação a $\mathrm{F} 1$. 
Adição de farinha de resíduos de guavira

TABELA 2: Composição físico-química média ( \pm desvio padrão) da farinha de resíduos de guavira (FRG) e das barras de cereais padrão (F1) e com adição de $8 \%$ de FRG (F5)

\begin{tabular}{lccccc}
\hline Parâmetro & FRG & F1 & VD $(\%)^{*}$ & F5 & VD $(\%)^{*}$ \\
\hline Umidade $\left(\mathrm{g} \cdot 100 \mathrm{~g}^{-1}\right)$ & $4,02 \pm 0,05$ & $10,60 \pm 0,07^{\mathrm{a}}$ & ND & $8,40 \pm 0,08^{\mathrm{b}}$ & ND \\
Cinzas $\left(\mathrm{g} \cdot 100 \mathrm{~g}^{-1}\right)$ & $2,27 \pm 0,02$ & $1,48 \pm 0,04^{\mathrm{b}}$ & $\mathrm{ND}$ & $1,58 \pm 0,03^{\mathrm{a}}$ & $\mathrm{ND}$ \\
Proteína $\left(\mathrm{g} \cdot 100 \mathrm{~g}^{-1}\right)$ & $6,26 \pm 0,09$ & $9,07 \pm 0,05^{\mathrm{a}}$ & 8,56 & $7,79 \pm 0,05^{\mathrm{b}}$ & 7,34 \\
Lipídio $\left(\mathrm{g} \cdot 100 \mathrm{~g}^{-1}\right)$ & $11,26 \pm 0,08$ & $11,03 \pm 0,09^{\mathrm{b}}$ & $\mathrm{ND}$ & $13,19 \pm 0,07^{\mathrm{a}}$ & $\mathrm{ND}$ \\
Carboidrato $\left(\mathrm{g} \cdot 100 \mathrm{~g}^{-1}\right)^{* *}$ & $76,19 \pm 0,56$ & $67,81 \pm 0,22^{\mathrm{b}}$ & 13,04 & $69,05 \pm 0,31^{\mathrm{a}}$ & 13,27 \\
Valor calórico total $\left(\mathrm{kcal}^{\mathrm{b}} 100 \mathrm{~g}^{-1}\right)$ & $431,59 \pm 0,98$ & $406,15 \pm 0,89^{\mathrm{b}}$ & 5,25 & $424,32 \pm 0,99^{\mathrm{a}}$ & 5,49 \\
Fibra solúvel $\left(\mathrm{g} \cdot 100 \mathrm{~g}^{-1}\right)^{* * *}$ & $5,78 \pm 0,10$ & $2,84 \pm 0,09^{\mathrm{b}}$ & $\mathrm{ND}$ & $3,42 \pm 0,08^{\mathrm{a}}$ & $\mathrm{ND}$ \\
Fibra insolúvel $\left(\mathrm{g} \cdot 100 \mathrm{~g}^{-1}\right)^{* * *}$ & $49,07 \pm 0,09$ & $5,27 \pm 0,10^{\mathrm{b}}$ & $\mathrm{ND}$ & $9,94 \pm 0,13^{\mathrm{a}}$ & $\mathrm{ND}$ \\
Fibra total $\left(\mathrm{g} \cdot 100 \mathrm{~g}^{-1}\right)^{* * * *}$ & $54,85 \pm 0,11$ & $8,12 \pm 0,12^{\mathrm{b}}$ & 7,59 & $13,36 \pm 0,15^{\mathrm{a}}$ & 12,49 \\
\hline
\end{tabular}

Letras distintas na linha indicam diferença significativa pelo teste de $\mathrm{t}$ de student ( $<00,05)$; *VD: nutrientes avaliados pela média da $\mathrm{DRI}^{29}$, com base numa dieta de $1.933,5 \mathrm{kcal} /$ dia e porção média de 25 gramas de barra de cereal (uma unidade); Valores calculados em base úmida; ${ }^{* *}$ Inclui fibra alimentar; ${ }^{* * *}$ Fibra alimentar; ND: não disponível. Fonte: os autores.

\section{Discussão}

A adição de até $8 \%$ de FRG em barra de cereais garante a aceitabilidade geral do produto por julgadores infantis. Resultados similares foram observados após a adição de resíduos de maracuja ${ }^{30} \mathrm{e}$ de abacaxi ${ }^{8}$ em barra de cereais. A menor aceitação da barra de cereais contendo maiores teores de FRG é explicada, provavelmente, devido ao forte aroma cítrico e sabor azedo que a guavira apresent $\mathrm{a}^{31}$. Além disso, a FRG conferiu coloração mais amarronzada às barras de cereais, devido ao escurecimento não enzimático (Reação de Maillard), processo que ocorre durante a desidratação da farinha. Efeito similar foi observado por Carvalho e Conti-Silva ${ }^{32}$ ao estudarem barra de cereais adicionada de diferentes níveis de farinha de casca de banana. Outros componentes presentes na guavira, como a vitamina $\mathrm{C}$ e os carotenoides, também sofrem modificações químicas pela ação do calor e têm sua coloração alterada ${ }^{33}$.

Durante a elaboração da barra de cereais verificouse que a massa das formulações com maiores teores de FRG tinham um aspecto mais quebradiço, apesar das crianças não terem percebido diferença na textura do produto. Segundo Carvalho e Conti-Silva ${ }^{32}$, a adição de ingredientes fibrosos, como farinha de cascas ou de resíduos de frutas, podem influenciar na textura dos alimentos em geral, aumentando a dureza, a mastigabilidade e a adesividade. Além disso, a adição de FRG reduz o teor de glúten do produto, pela redução na concentração de farinha de trigo. Isso contribui com a redução da maciez, uma vez que o glúten é uma proteína responsável pela retenção de gases da fermentação e crescimento da massa ${ }^{34,35}$. Apesar das menores notas obtidas após a adição de FRG, as formulações apresentaram IA > 70\% em todos os atributos, o que demonstra uma boa aceitação sensorial ${ }^{25}$. Esses resultados corroboram Zanelato et $a l .^{36}$, que estudaram barra de cereais elaborada com farinha de casca de pequi $(5 \%)$. Cristo et al. $^{37}$, ao realizar estudo com barra de cereais adicionadas $(0 \mathrm{a}$ $27 \%$ ) de resíduos de casca de chuchu, encontraram resultados semelhantes àqueles observados na FIGURA 1. Dessa forma, demonstra-se a viabilidade da utilização de farinha de resíduos de frutas, como a guavira, como ingrediente em produtos alimentícios. Essa estratégia colabora para o aproveitamento de partes não convencionais de alimentos e como ferramenta para o incremento de nutrientes na dieta habitual de crianças.

O teor de umidade da FRG está de acordo a Resolução $\mathrm{n}^{\mathrm{o}} 263$ de 22 de setembro de $2005^{38}$, que recomenda um valor máximo de umidade de $15 \%$ para cereais, amidos, farinhas e farelos. Com isso, possível prolongar a vida útil e aumentar a estabilidade microbiológica durante o armazenamento do produto $^{39}$. O elevado teor de cinzas verificado na FRG é atribuído à presença de minerais como, por exemplo, o potássio $\left(257,47 \mathrm{mg} .100 \mathrm{~g}^{-1}\right)$, o cálcio $(31,53$ $\left.\mathrm{mg} \cdot 100 \mathrm{~g}^{-1}\right)$ e o ferro $\left(1,08 \mathrm{mg} \cdot 100 \mathrm{~g}^{-1}\right)^{40}$. De forma similar, o considerável teor de fibra presente na FRG torna esse produto recomendável para adição em produtos alimentícios como forma de enriquecimento nutricional $^{41}$.

Os teores de umidade e de proteína constatados em F1 e F5 estão de acordo com aqueles observados em um estudo que avaliou a adição (5 a 20\%) de farinha de casca de laranja em biscoitos ${ }^{42}$. Isso pode ser explicado, principalmente, porque as proteínas possuem capacidade de absorver seu próprio peso em água. Embora tenha sido observada diminuição no teor de proteína na barra de cereais com adição de $8 \%$ de FRG, o produto ainda constitui uma importante fonte deste nutriente ${ }^{43}$, é adequado como complemento alimentar para o público infantil. As demais diferenças observadas se devem aos menores conteúdos de cinzas $\left(0,8\right.$ g. $\left.100 \mathrm{~g}^{-1}\right)$, lipídio $\left(0,98 \mathrm{~g} \cdot 100 \mathrm{~g}^{-1}\right)$, carboidrato

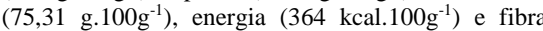
alimentar total $\left(2,7 \mathrm{~g} .100 \mathrm{~g}^{-1}\right)^{44}$ presentes na farinha de trigo, quando comparada à FRG (TABELA 2). O teo de fibra alimentar total verificado na formulação adicionada de $8 \%$ de FRG (F5) se deve, principalmente, ao elevado teor de fibras presente na 
Adição de farinha de resíduos de guavira

FRG $\left(54,85 \mathrm{~g} \cdot 100 \mathrm{~g}^{-1}\right)$. Contudo, as amostras F1 e F5 podem ser consideradas produtos com alto teor de fibra alimentar ${ }^{43}$. Pesquisas já confirmaram a importância do consumo de fibras para o público infantil, já que apresentam propriedades para o adequado funcionamento intestinal e auxiliam a reduzir o risco de doenças crônicas futuras ${ }^{45,46}$. Diante disso, recomenda-se a ingestão de barra de cereais adicionada de FRG por crianças em fase escolar.

\section{Conclusão}

Um nível de adição de até $8 \%$ de FRG em barra de cereais é bem aceito pelas crianças, obtendo-se aceitação sensorial semelhante ao produto padrão. Além disso, proporcionou aumento nos teores minerais e fibras, foi considerado um produto de alto conteúdo de fibras alimentares. A FRG também pode ser classificada como fonte de proteína, nutriente essencial na nutrição infantil. Assim, torna-se um potencial ingrediente para adição em barra de cereais e produtos similares, com possibilidade de ser oferecida ao público infantil e com altas expectativas de comercialização. Também, colabora para a redução do desperdício alimentar e dos efeitos negativos do descarte de lixo orgânico no meio ambiente.

\section{Declaração de conflitos de interesses}

Os autores do artigo afirmam que não houve nenhuma situação de conflito de interesse, tais como propostas de financiamento, emissão de pareceres, promoções ou participação em comitês consultivos ou diretivos, entre outras, que pudessem influenciar no desenvolvimento do trabalho.

\section{Referências}

1. ORGANIZACIÓN DE LAS NACIONES UNIDAS PARA LA ALIMENTACIÓN Y LA AGRICULTURA (FAO). Pérdidas y desperdícios de alimentos em América Latina y el Caribe. 2016. Disponível em:<http://www.fao.org/3/a-i5504s.pdf $>$. Acesso em: 26 de outubro de 2018 .

2. KOWALSKA, H.; CZAJKOWSKA, K.; CICHOWSKA, J.; LENART, A. What's new in biopotential of fruit and vegetable by-products applied in the food processing industry. Trends in Food Science \& Technology, Cambridge, v.67, n.1, p.150159, 2017.

3. KUMAR, K * YADAV, A. N. KUMAR, V; VYAS, P.; DHALIWAL, H. S. Food waste: a potential bioresource for extraction of nutraceuticals and bioactive compounds. Bioresources and

Bioprocessing, London, v. 4, n. 1, p. 18, 2017.

4. SOCACI, S. A.; FĂRCAŞ, A. C.; VODNAR, D. C.; TOFANA, M. Food wastes as valuable sources of bioactive molecules. Superfood and Functional Food The Development of Superfoods and Their Roles as Medicine. Rijeka, v.1, n.1, p. 75-93, 2017.
5. SAGAR, N. A.; PAREEK, S.; SHARMA, S YAHIA, E. M.; LOBO, M. G. Fruit and vegetable waste: Bioactive compounds, their extraction, an possible utilization. Comprehensive Reviews in Food Science and Food Safety, Chicago, v. 17, n. 3 , p. $512-531,2018$

6. BERTAGNOLLI, S. M. M.; SILVEIRA, M. L. R.; FOGACA, A. D. O.; UMANN, L.; PENNA, N. G Bioactive compounds and acceptance of cookies made with Guava peel flour. Food Science and Technology, Campinas, v.34, n.2, p.303-308, 2014.

7. OLIVEIRA, D. M.; MARQUES, D. R KWIATKOWSKI, A.; MONTEIRO, A. R. G. CLEMENTE, E. Sensory analysis and chemical characterization of cereal enriched with grape peel and seed flour. Acta Scientiarum Technology, Maringá, v. 35, n. 3, p. $427-431,2013$.

8. DAMASCENO, K. A.; GONCALVES, C. A. A.; PEREIRA, G. S.; COSTA, L. L.; CAMPAGNOL, P C. B.; ALMEIDA, P. L.; PEREIRA, L. A Development of Cereal Bars Containing Pineapple Peel Flour (Ananas comosus L. Merril). Journal o Food Quality, United Kingdom, v.39, n.5, p.417-424, 2016.

9. BREDA, C. A.; SANJINEZ-ARGANDOÑA, E. J CORREIA, C. A. C. Shelf life of powdered Campomanesia adamantium pulp in controlled environments. Food Chemistry, Barking, v.135, n.4 p.2960-2964, 2012.

10. VISCARDI, D. Z.; OLIVEIRA, V. S. D.; ARRIGO, J. D. S.; PICCINELLI, A. C.; CARDOSO, C. A.; MALDONADE, I. R.; KASSUYA C. A L.;SANJINEZ-ARGANDOÑA, E. J. Antiinflammatory, and antinociceptive effects of Campomanesia adamantium microencapsulated pulp. Brazilian Journal of Pharmacognosy, São Paulo, v.27, n.2, p.220-227, 2017.

11. FERREIRA, D. F; GARRUTI, D. S. BARIN, J. S.; CICHOSKI, A. J.; WAGNER, R. Characterization of Odor-Active Compounds in Gabiroba Fruits (Campomanesia xanthocarpa O. Berg). Journal of Food Quality, United Kingdom, v.39, n.2, p.90-97, 2015.

12. MELO, R. M. Caracterização dos atributos químicos de substratos e do desenvolvimento de mudas de Campomanesia adamantium (cambess.) o. berg em resposta à calagem e caracterização nutricional do fruto nativo e do licor elaborado. 2017. 80 p. Dissertação (Mestrado em Agronomia Produção vegetal) - Universidade Federal da Grande Dourados, Dourados, 2017.

13. CRISTOFEL, C. J. Elaboração de hambúrguer de tilápia (oreochromis niloticus) enriquecido com ingrediente funcional e resíduo de guabiroba (Campomanesia xanthocarpa): caracteristicas físicas, químicas e sensoriais. 2014. 77 p. Trabalho de Conclusão de Curso (Graduação em Engenharia de alimentos) - Universidade Federal da Fronteira do Sul. Laranjeiras do Sul, 2014

14. MENDES, R. M.; PINTO, E. G.; SOARES, D. B.

$S$. Determinação dos compostos bioativos da 
Adição de farinha de resíduos de guavira

gabiroba. Agrarian, Dourados, v.11, n.39, p.68-72, 2018

15. LOBATO, L. P.; PEREIRA, A. E. I. C.; LAZARETTI, M. M.; BARBOSA, D. S.; CARREIRA, C. M.; MANDARINO, J. M. G.; GROSSMANN, M. V. E. Snack bars with high soy protein and isoflavone content for use in diets to control dyslipidaemia. International Journal of Food Sciences and Nutrition, Basingstoke, v.63, n.1, p.49-58, 2012

16. CUNHA, F. C.; SIQUEIRA, I. F.; PELEGRIN, J. O.; SOUZA, A. V. O. M.; VASCONCELOS, M. P.; JORDÃO, C.; SANTOS, E. F.; HIANE, P. A.; SANCHES, F. L. F. Z. Development of cereal bars using bacuri pulp flour for sportsmen: nutritional composition and sensory acceptability. International Journal of Development Research, Tamil Nadu, v.8, n.2, p.18947-18953, 2018

17. MATUK, T. T.; STANCARI, P. C. S.; BUENO, M. B.; ZACARELLI, E. M. Composição de lancheiras de alunos de escolas particulares de São Paulo. Revista Paulista de Pediatria, São Paulo, v. 29, n. 2, p. 157-163, 2011.

18. SANIGORSKI, A.M; BELL, A.C; KREMER, P.J.; SWINBURN, B.A. Lunchbox contents of Australian school children: room for improvement. European Journal of Clinical Nutrition, London, v.59, n.11, p.1310-1316, 2015. 19. SREBERNICH, S. M.; GONÇALVES, G. M. S., ORMENESE, R. D. C. S. C., \& RUFFI, C. R. G. Physico-chemical, sensory and nutritional characteristics of cereal bars with addition of acacia gum, inulin and sorbitol. Food Science and Technology, London, v. 36, n. 3, p. 555-562, 2016. 20. CARVALHO, C. A.; FONSÊCA, P. C. A.; PIORE, S. L.; FRANCESCHINI, S. C. C.; NOVAES, J. F. Consumo alimentar e adequação nutricional em crianças brasileiras: revisão sistemática. Revista Paulista de Pediatria, São Paulo, v.33, n.2, p.211221, 2015.

21. ATZINGEN, M. C. B. C.; SILVA, M. E. M P. Sensory characteristics of food as a determinant of food choices. Nutrire-Revista da Sociedade Brasileira de Alimentação e Nutrição, São Paulo, v. 35, n. 3, p. 183-196, 2010.

22. BRITO, A. L. B.; BRITO, L. R.; HONORATO, F. A.; PONTES, M. J. C.; PONTES, L. F. B. L. Classification of cereal bars using near infrared spectroscopy and linear discriminant analysis. Food Research International, Essex, v.51, n.2, p.924-928, 2013

23. DUTCOSKY, S. D. Análise sensorial de alimentos. 4. ed. Curitiba: Champagnat, 2013.

24. BRASIL. Ministério da Educação. Fundo Nacional de Desenvolvimento da Educação. Resolução $n^{\circ} 26$ de 17 de junho de 2013. Dispõe sobre o atendimento da alimentação escolar aos alunos da educação básica no âmbito do Programa Nacional de Alimentação Escolar - PNAE. Diário Oficial da União, Brasília, DF, 18 jun. 2013.
25. TEIXEIRA, E.; MEINERT, E.; BARBETTA, P. A. Análise sensorial dos alimentos. Florianópolis: UFSC, 1987.

26. BLIGH, E. G.; DYER, W. J. A rapid method of total lipid extraction and purification. Canadian Journal of Biochemistry and Physiology, Ottawa, v.37, n.8, p.911-917, 1959

27. ASSOCIATION OF OFFICIAL ANALYTICAL CHEMISTRY (AOAC). Official methods of analysis of AOAC international. 20. ed. Gaithersburg: AOAC, 2016.

28. MERRILL, A. L.; WATT, B. K. Energy values of foods: basis and derivation. Washington: United States Department of Agriculture Handbook, 1973.

29. DIETARY REFERENCE INTAKES (DRI) Dietary reference intakes for energy, carbohydrate, fiber, fat, fatty acids, cholesterol, protein, and amino acids (macronutrients) Washington: The National Academies Press, 2005.

30. SILVA, I. Q.; OLIVEIRA, B. D.; LOPES, A. S. PENA, R. D. S. Obtenção de barra de cereais adicionada do resíduo industrial de maracujá. Alimentos e Nutrição, Araraquara, v.20, n.2, p.321-329, 2009.

31. FERNANDES, T. O.; ÁVILA, R. I.; MOURA, S S.; RIBEIRO, G. A.; NAVES, M. M. V.; VALADARES, M. C. Campomanesia adamantium (Myrtaceae) fruits protect HEPG2 cells against carbon tetrachloride-induced toxicity. Toxicology Reports, Heraklion, v.2, n.1, p.184-193, 2015.

32. CARVALHO, V. S.; CONTI-SILVA, A. C. Cerea bars produced with banana peel flour: evaluation of acceptability and sensory profile. Journal of the Science of Food and Agriculture, Oxford, v.98, n.1, p.134-139, 2017.

33. HIANE, P. A.; BOGO, D; RAMOS, M. I. L. RAMOS FILHO, M. M. Carotenoides pró-vitamínicos A e composição em ácidos graxos do fruto e da farinha do bacuri (Scheelea phalerata Mart.). Ciência e Tecnologia de Alimentos, Campinas, v.2, n.23, p.206-209, 2003.

34. TULYATHAN, V.; TANANUWONG, K. SONGJINDA, P.; JAIBOON, N. Some physicochemical properties of jackfruit (Artocarpus heterophyllus Lam) seed flour and starch. Science Asia, Bankok, v.28, n.1, p.37-41, 2002.

35. HAMADA, S.; SUZUKI, K.; AOKI, N.; SUZUKI Y. Improvements in the qualities of gluten-free bread after using a protease obtained from Aspergillus oryzae. Journal of Cereal Science, London, v.57, n.1, p.91-97, 2013.

36. ZANELATO, E F N ; VIANA, E S. M ESPESCHIT, A. C.; CARDOSO, L. M.; VIANA, A O. Análise sensorial de uma barra de cereal preparada com farinha da casca de pequi (Caryocar brasiliense Camb.) Revista Científica Univiçosa, Viçosa, v.8, n.1, p.104-109, 2016.

37. CRISTO, T. W.; RODRIGUES, B. M.; DOS SANTOS, N. M.; CANDIDO, C. J.; DOS SANTOS, E. F.; NOVELLO, D. Barra de cereais com adição de 
farinha de casca de chuchu: caracterização físicoquímica e sensorial entre crianças. Semina: Ciências Biológicas e da Saúde, Londrina, v. 36, n. 2, p. 85-96, 2015

38. BRASIL. Ministério da Saúde. Agência Nacional de Vigilância Sanitária (ANVISA). Resolução nº 263, de 22 de setembro de 2005. Regulamento técnico para produtos de cereais, amidos, farinhas e farelos. 2005. Diário Oficial União, Poder Executivo. Brasília, DF, 2005.

39. SREBERNICH, S. M.; MEIRELES, F.; LOURENÇÃO, G. Avaliação microbiológica de barras de cereais diet por meio de agente ligante colágeno hidrolisado e goma acácia. Revista de Ciências Médicas, Campinas, v.20, n.1/2, p.5-13, 2011.

40. ALVES, A. M.; ALVES, M. S. O.; FERNANDES, T. O.; NAVES, R. V.; NAVES, M. M. V. Caracterização física e química, fenólicos totais e atividade antioxidante da polpa e resíduo de gabiroba. Revista Brasileira de Fruticultura, Cruz das Almas, v.35, n.3, p.837-844, 2013.

41. SOUZA, J. C.; PICCINELLI, A. C.; AQUINO, D. F. SOUZA, V. V; SCHMITZ, W O.; TRAESEL, G. K.; CARDOSO, A. L.; KASSUYA, C. A. L.; ARENA, A. C. Toxicological analysis and antihyperalgesic, antidepressant, and anti-inflammatory effects of Campomanesia adamantium fruit barks. Nutritional Neuroscience, Amsterdam, v.20, n.1, p.23-31, 2017. 42. OBAFAYE, R. O.; OMOBA, O. S. Orange peel flour: A potential source of antioxidant and dietary fiber in pearl-millet biscuit. Journal of Food Biochemistry, Voorhees, e12523, p.1-8, 2018.

43. BRASIL. Ministério da Saúde. Agência Nacional de Vigilância Sanitária (ANVISA). RDC nº. 54, de 12 de novembro de 2012. Regulamento técnico sobre Informação Nutricional. 2012. Diário Oficial União, Poder Executivo. Brasília, DF, 2012

44. UNITED STATES DEPARTMENT OF AGRICULTURE (USDA). Agricultural Research Service. Food Composition Databases. 2018. Disponível em: <https://ndb.nal.usda.gov/ndb/foods/show/305354?m anu $=\& \mathrm{fgcd}=\& \mathrm{ds}=\& \mathrm{q}=$ Wheat $\% 20$ flour $\% 20$ white,$\% 2$ 0all-purpose, \%20unenriched>. Acesso em: $04 \mathrm{de}$ outubro de 2018

45. ANDERSON, J. W.; BAIRD, P.; DAVIS, R. H JR.; FERRERI, S.; KNUDTSON, M.; KORAYM, A.; WATERS, V.; WILLIAMS, C. L. Health benefits of dietary fiber. Nutrition Reviews, New York, v.67, n. 4, p. 188-205, 2009

46. KRANZ, S.; BRAUCHLA, M.; SLAVIN, J. L.; MILLER, K. B. What do we know about dietary fiber intake in children and health? The effects of fiber intake on constipation, obesity, and diabetes in children. Advances in Nutrition, Bethesda, v.3, n.1, p.47-53, 2012. 